\title{
ESTIMATES OF THE TRANSITION DENSITIES FOR THE REFLECTED BROWNIAN MOTION ON SIMPLE NESTED FRACTALS*
}

\author{
BY
}

MARIUSZ OLSZEWS K I (WROCŁAW)

\begin{abstract}
We give sharp two-sided estimates for the functions $g_{M}(t, x, y)$ and $g_{M}(t, x, y)-g(t, x, y)$, where $g_{M}(t, x, y)$ are the transition probability densities of the reflected Brownian motion on an $M$ complex of order $M \in \mathbb{Z}$ of an unbounded planar simple nested fractal and $g(t, x, y)$ are the transition probability densities of the "free" Brownian motion on this fractal. This is done for a large class of planar simple nested fractals with the good labeling property.
\end{abstract}

2010 AMS Mathematics Subject Classification: Primary: 60J35, 28A80; Secondary: 60J25, 60J65.

Key words and phrases: Projection, good labeling property, reflected process, transition probability density, simple nested fractal, graph metric, Sierpiński gasket.

\section{INTRODUCTION}

The analysis and probability theory (especially stochastic processes) on fractals underwent rapid development over the last decades (see e.g. [1], [12], [23], [24] and the references therein). The original motivation came from the investigations on the properties of disordered media in mathematical physics. Fractals also help us to understand the features of natural phenomena such as polymers, and growth of molds and crystals. The rigorous definition of the Brownian motion on the Sierpiński gasket has been given by Barlow and Perkins in [3] (see also [7], [17]). Lindstrøm [19] used a nonstandard analysis to construct such a process on general simple nested fractals (see also [6], [18], [22] for a Dirichlet form approach). The case of more general fractals was also addressed in [2], [14], [16]. The estimates of the transition densities for the Brownian motion on simple nested fractals were proved by Kumagai in [13]. The case of a more general class of finitely ramified fractals (called affine nested fractals) was studied in [5].

${ }^{*}$ Research supported by the National Science Center, Poland, grant no. 2015/17/B/ST1/01233. 
The present article is a companion paper to [9], where the reflected Brownian motion in an $M$-complex, $M \in \mathbb{Z}$, of a simple nested fractal was constructed (see also the paper [20] for the case of the Sierpiński triangle). Such a process was obtained as a "folding" projection of the free Brownian motion from the unbounded fractal and the whole construction was performed under the key geometric assumption that the fractal has a good labeling property (see Section 2 for more details). It was also proved in the cited paper that the one-dimensional distributions of this process have the continuous and symmetric densities $g_{M}(t, x, y)$ (see (2.9) for a definition), which provided us with further regularity properties of the reflected process.

Our main goal in this paper is to find the sharp estimates of the densities $g_{M}(t, x, y)$ and for differences $g_{M}(t, x, y)-g(t, x, y)$, where $g(t, x, y)$ are the transition probability densities of a "free" process. We give an argument which allows us to deduce the two-sided sharp estimates for these functions from the intrinsic growth property of the graph metric on the planar nested fractal (for the definition of the graph metric $d_{M}(x, y)$ see (2.4)). More precisely, we show that there exist positive constants $c_{1}, \ldots, c_{6}$ (uniform in $t, x, y$ and $M$ ) such that

$$
\begin{aligned}
c_{1}\left(f_{c_{2}}(t,|x-y|) \vee h_{c_{3}}(t, M)\right) & \leqslant g_{M}(t, x, y) \\
& \leqslant c_{4}\left(f_{c_{5}}(t,|x-y|) \vee h_{c_{6}}(t, M)\right),
\end{aligned}
$$

where

$$
f_{c}(t, r)=t^{-d_{f} / d_{w}} \exp \left(-c\left(\frac{r^{d_{w}}}{t}\right)^{1 /\left(d_{J}-1\right)}\right)
$$

and

$h_{c}(t, M)=L^{-d_{f} M}\left(\frac{L^{M}}{t^{1 / d_{w}}} \vee 1\right)^{d_{f}-d_{w} /\left(d_{J}-1\right)} \exp \left(-c\left(\frac{L^{M}}{t^{1 / d_{w}}} \vee 1\right)^{d_{w} /\left(d_{J}-1\right)}\right)$.

This result is given in Theorem B.J. Here $L$ is a scaling factor of the fractal and $d_{f}, d_{w}$ and $d_{J}$ are certain parameters determined by the geometry of the fractal. One can see from the above estimates that for $t \geqslant L^{M d_{w}}$ the density $g_{M}(t, x, y)$ behaves like $L^{-M d_{f}}$. If $t<L^{M d_{w}}$, then the reflected process less "feels" the reflection and resembles the free diffusion (see Corollary B.] for details). This effect is explained by our second main result (Theorem B.2), which gives the sharp twosided estimates for the difference $g_{M}(t, x, y)-g(t, x, y)$. Indeed, we prove that there are positive constants $c_{7}, \ldots, c_{12}$ (again uniform in $t, x, y$ and $M$ ) such that

$$
\begin{aligned}
c_{7}\left(f_{c_{8}}\left(t, \delta_{M}(x, y)\right) \vee h_{c_{9}}(t, M)\right) & \leqslant g_{M}(t, x, y)-g(t, x, y) \\
& \leqslant c_{10}\left(f_{c_{11}}\left(t, \delta_{M}(x, y)\right) \vee h_{c_{12}}(t, M)\right),
\end{aligned}
$$

where $\delta_{M}(x, y)=\inf _{z \in V_{M}^{*}}(|x-z|+|z-y|)$ and $V_{M}^{*}$ is the set of all vertices of a basic $M$-complex that connect it with other $M$-complexes. By Corollary B.] 
mentioned above, this results describes how the dependence on the boundary of the complex emerges in the distribution of the reflected process.

We would like to emphasize that we have direct applications for the estimates obtained in the present paper. In the articles [10], [1]], the reflected Brownian motion was used to prove the existence and further asymptotic properties of the integrated density of states for subordinate Brownian motions evolving in presence of the Poissonian random field on the Sierpinski triangle. The estimates of the densities were an essential tool there. The present results will allow us to continue this research in the case when the configuration space is modeled by a general simple nested fractal. In this context, it is crucial that our estimates describe the behavior of $g_{M}(t, x, y)$ not only in $x, y$ and $t$, but also in $M$. This is the subject of our ongoing project.

At the end of the Introduction, let us say a few words about our methods. First note that our upper bound for the tail of the series in Lemma 3.5 extends a similar result in [10], Lemma 2.5, obtained for the reflected Brownian motion on the Sierpiński gasket. The proof of that bound in an essential way uses the facts that the $M$-complexes of any order $M \in \mathbb{Z}$ of the gasket agree with the Euclidean balls $B\left(0,2^{M}\right)$ intersected with the fractal and that the geodesic (or the shortest path) metric is uniformly comparable to the Euclidean one. Such a comparability condition is also a common assumption in the papers dealing with subordinate Brownian motions on fractals having the $d$-set structure, see [4], [8]. This argument does not have an extension to the general nested fractals, for which the geodesic metric is typically not well defined. To overcome this difficulty, we propose a new approach based on an application of the graph metric of order $M$ that works well for all nested fractals. Our main contribution is the observation that the intrinsic growth property of the graph metric stated in Lemma B.J leads to the sharp estimates of densities $g_{M}(t, x, y)$. We also want to mention that the concluding part of the proof of the upper bound in Lemma 3.5 follows the general ideas from the proof of Lemma 2.5 in [10], while Lemma 3.3 is a new key observation.

\section{PRELIMINARIES}

2.1. Planar simple nested fractals. Consider a collection of similitudes $\Psi_{i}$ : $\mathbb{R}^{2} \rightarrow \mathbb{R}^{2}$ with a common scaling factor $L>1$ and a common isometry part $U$, i.e. $\Psi_{i}(x)=(1 / L) U(x)+\nu_{i}$, where $\nu_{i} \in \mathbb{R}^{2}, i \in\{1, \ldots, N\}$. We shall assume $\nu_{1}=0$, which will allow us to write $\mathcal{K}^{\langle M\rangle}=L^{M} \mathcal{K}^{\langle 0\rangle}$ without using the translation before or after scaling. There exists a unique nonempty compact set $\mathcal{K}^{\langle 0\rangle}$ (called the fractal generated by the system $\left.\left(\Psi_{i}\right)_{i=1}^{N}\right)$ such that $\mathcal{K}^{\langle 0\rangle}=\bigcup_{i=1}^{N} \Psi_{i}\left(\mathcal{K}^{\langle 0\rangle}\right)$. As $L>1$, each similitude has exactly one fixed point.

DEFINITION 2.1 (Essential fixed point). A fixed point $x \in \mathcal{K}^{\langle 0\rangle}$ is an essential fixed point if there exists another fixed point $y \in \mathcal{K}^{\langle 0\rangle}$ and two different similitudes $\Psi_{i}, \Psi_{j}$ such that $\Psi_{i}(x)=\Psi_{j}(y)$. 
The set of all essential fixed points of transformations $\Psi_{1}, \ldots, \Psi_{N}$ is denoted by $V_{0}^{\langle 0\rangle}$. Clearly, $k:=\# V_{0}^{\langle 0\rangle} \leqslant N$. For the Sierpiński gasket $k=N$, but there are many examples with $k<N$ (see Fig. 1).

DEFINITION 2.2 (Simple nested fractal). The fractal $\mathcal{K}^{\langle 0\rangle}$ generated by the system $\left(\Psi_{i}\right)_{i=1}^{N}$ is called a simple nested fractal (SNF) if the following five conditions are satisfied:

(1) $\# V_{0}^{\langle 0\rangle} \geqslant 2$.

(2) (Open set condition) There exists an open set $U \subset \mathbb{R}^{2}$ such that for $i \neq j$ we have $\Psi_{i}(U) \cap \Psi_{j}(U)=\emptyset$ and $\bigcup_{i=1}^{N} \Psi_{i}(U) \subseteq U$.

(3) (Nesting) $\Psi_{i}\left(\mathcal{K}^{\langle 0\rangle}\right) \cap \Psi_{j}\left(\mathcal{K}^{\langle 0\rangle}\right)=\Psi_{i}\left(V_{0}^{\langle 0\rangle}\right) \cap \Psi_{j}\left(V_{0}^{\langle 0\rangle}\right)$ for $i \neq j$.

(4) (Symmetry) For $x, y \in V_{0}^{\langle 0\rangle}$, denote by $S_{x, y}$ the symmetry with respect to the line bisecting the segment $[x, y]$. Then

$$
\forall i \in\{1, \ldots, M\} \forall x, y \in V_{0}^{\langle 0\rangle} \exists j \in\{1, \ldots, M\} S_{x, y}\left(\Psi_{i}\left(V_{0}^{\langle 0\rangle}\right)\right)=\Psi_{j}\left(V_{0}^{\langle 0\rangle}\right) .
$$

(5) (Connectivity) On the set $V_{-1}^{\langle 0\rangle}:=\bigcup_{i} \Psi_{i}\left(V_{0}^{\langle 0\rangle}\right)$ we define a graph structure $E_{-1}$ as follows: $(x, y) \in E_{-1}$ if and only if $x, y \in \Psi_{i}\left(\mathcal{K}^{\langle 0\rangle}\right)$ for some $i$. Then the $\operatorname{graph}\left(V_{-1}^{\langle 0\rangle}, E_{-1}\right)$ is required to be connected.

If $\mathcal{K}^{\langle 0\rangle}$ is a simple nested fractal, then we put

$$
\mathcal{K}^{\langle M\rangle}=L^{M} \mathcal{K}^{\langle 0\rangle}, \quad M \in \mathbb{Z},
$$

and

$$
\mathcal{K}^{\langle\infty\rangle}=\bigcup_{M=0}^{\infty} \mathcal{K}^{\langle M\rangle}
$$

The set $\mathcal{K}^{\langle\infty\rangle}$ is the unbounded simple nested fractal (USNF).

Definition 2.3. Let $M \in \mathbb{Z}$. Then define:

(1) An $M$-complex: every set $\Delta_{M} \subset \mathcal{K}^{\langle\infty\rangle}$ of the form

$$
\Delta_{M}=\mathcal{K}^{\langle M\rangle}+\sum_{j=M+1}^{J} L^{j} \nu_{i_{j}}
$$

for some $J \geqslant M+1, \nu_{i_{j}} \in\left\{\nu_{1}, \ldots, \nu_{N}\right\}$, is called an $M$-complex. The set of all $M$-complexes in $\mathcal{K}^{\langle\infty\rangle}$ is denoted by $\mathcal{T}_{M}$.

(2) Vertices of an $M$-complex: the set $V\left(\Delta_{M}\right)=L^{M} V_{0}^{\langle 0\rangle}+\sum_{j=M+1}^{J} L^{j} \nu_{i_{j}}$. (3) Vertices of $\mathcal{K}^{\langle M\rangle}$ :

$$
V_{M}^{\langle M\rangle}=V\left(\mathcal{K}^{\langle M\rangle}\right)=L^{M} V_{0}^{\langle 0\rangle} .
$$


(4) Vertices of all $M$-complexes inside an $(M+m)$-complex for $m>0$ :

$$
V_{M}^{\langle M+m\rangle}=\bigcup_{i=1}^{N} V_{M}^{\langle M+m-1\rangle}+L^{M} \nu_{i}
$$

(5) Vertices of all 0-complexes inside the unbounded nested fractal:

$$
V_{0}^{\langle\infty\rangle}=\bigcup_{M=0}^{\infty} V_{0}^{\langle M\rangle} .
$$

(6) Vertices of $M$-complexes from the unbounded fractal:

$$
V_{M}^{\langle\infty\rangle}=L^{M} V_{0}^{\langle\infty\rangle}
$$

(7) The unique $M$-complex containing $x \in \mathcal{K}^{\langle\infty\rangle} \backslash V_{M}^{\langle\infty\rangle}$ is denoted by $\Delta_{M}(x)$.

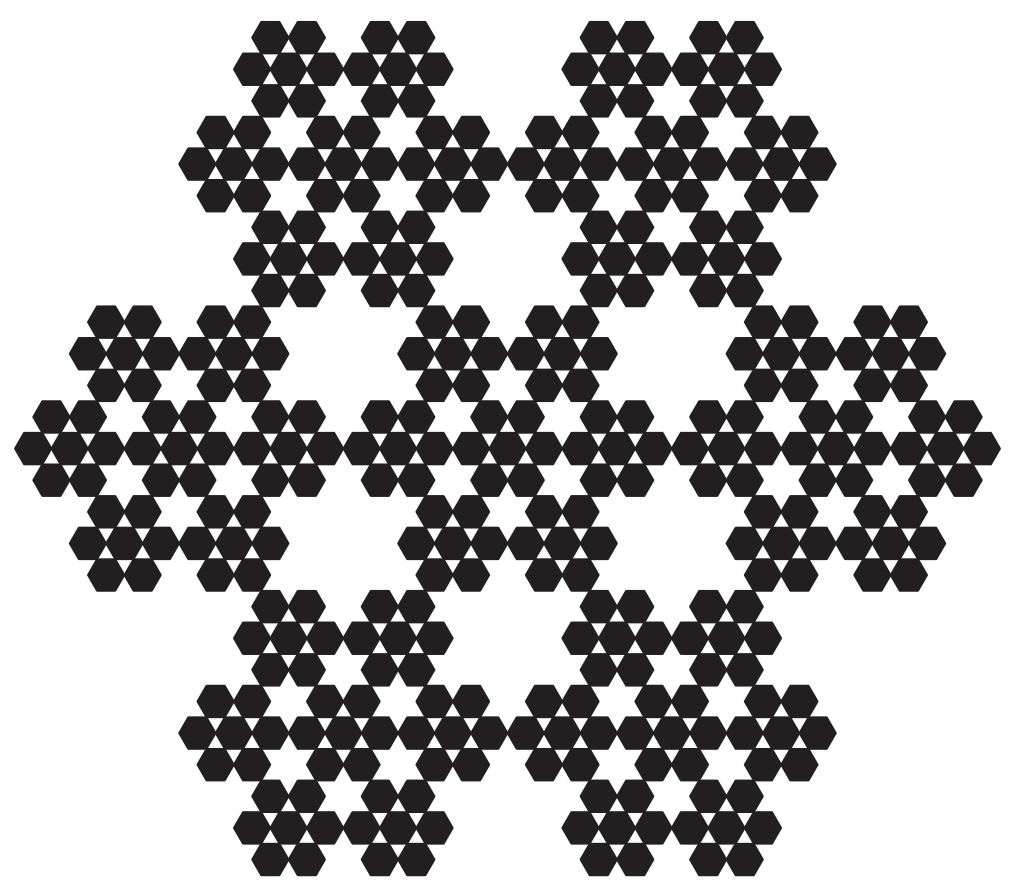

FigURE 1. An example of a nested fractal: the Lindstrøm snowflake. It is constructed by seven similitudes with $L=3$. It has seven fixed points, but only six essential fixed points.

By $d_{f}, d_{w}$ and $d_{s}$ we denote the Hausdorff dimension, the walk dimension and the spectral dimension of the SNF $\mathcal{K}^{\langle 0\rangle}$, respectively. It is known that the identity $d_{f} / d_{w}=d_{s} / 2$ holds. 
The $M$-graph metric on $\mathcal{K}^{\langle\infty\rangle} \times \mathcal{K}^{\langle\infty\rangle}$ is defined as follows:

$$
d_{M}(x, y):= \begin{cases}0 & \text { if } x=y ; \\ 1 & \text { if there exists } \Delta_{M} \in \mathcal{T}_{M} \text { such that } x, y \in \Delta_{M} ; \\ n>1 & \text { if there does not exist } \Delta_{M} \in \mathcal{T}_{M} \text { such that } x, y \in \Delta_{M} \\ & \text { and } n \text { is the lowest number for which there exist } \\ & \Delta_{M}^{(1)}, \Delta_{M}^{(2)}, \ldots, \Delta_{M}^{(n)} \in \mathcal{T}_{M} \text { such that } x \in \Delta_{M}^{(1)}, \\ & y \in \Delta_{M}^{(n)} \text { and } \Delta_{M}^{(i)} \cap \Delta_{M}^{(i+1)} \neq \emptyset \text { for } 1 \leqslant i \leqslant n-1 .\end{cases}
$$

Less formally, the $M$-graph metric means the smallest number of $M$-complexes the process must walk through when passing from $x$ to $y$.

2.2. Good labeling property and folding projections. Throughout this section we assume that $M \in \mathbb{Z}$ is arbitrary but fixed. Note that every $M$-complex $\Delta_{M}$ is a regular polygon with $k$ vertices (see [9], Proposition 2.1). In consequence, there exist exactly $k$ different rotations $R_{i}$ around the barycenter of $\mathcal{K}^{\langle M\rangle}$, mapping $\mathcal{K}^{\langle M\rangle}$ onto $\mathcal{K}^{\langle M\rangle}$ (for $i=1,2, \ldots, k$ the rotation $R_{i}$ rotates by the angle $2 \pi i / k$ ). Put $\mathcal{R}_{M}=\left\{R_{1}, \ldots, R_{k}\right\}$.

The concept of the good labeling property (GLP in short) has been introduced in [9]. Given the set of labels $\mathcal{A}=\left\{a_{1}, \ldots, a_{k}\right\}$, the labeling function is a map $\ell_{M}: V_{M}^{\langle\infty\rangle} \rightarrow \mathcal{A}$. It provides a good labeling (of order $M$ ) if every $M$-complex has the complete set of labels mapped to its vertices and the vertices of any $M$ complex are labeled in the same orientation. More precisely:

(1) For every $M$-complex $\Delta_{M}$ the restriction of $\ell_{M}$ to $V\left(\Delta_{M}\right)$ is a bijection onto $\mathcal{A}$.

(2) For every $M$-complex $\Delta_{M}$ of the form

$$
\Delta_{M}=\mathcal{K}^{\langle M\rangle}+\sum_{j=M+1}^{J} L^{j} \nu_{i_{j}}
$$

with some $J \geqslant M+1$ and $\nu_{i_{j}} \in\left\{\nu_{1}, \ldots, \nu_{N}\right\}$ (cf. Definition 2.3$]$ (1)), there exists a rotation $R_{\Delta_{M}} \in \mathcal{R}_{M}$ such that

$$
\ell_{M}(v)=\ell_{M}\left(R_{\Delta_{M}}\left(v-\sum_{j=M+1}^{J} L^{j} \nu_{i_{j}}\right)\right), \quad v \in V\left(\Delta_{M}\right) .
$$

The fractal $\mathcal{K}^{\langle\infty\rangle}$ is said to have the GLP if for some $M \in \mathbb{Z}$ there exists a labeling function $\ell_{M}$ satisfying both the conditions above. Note that due to the self-similarity of this set, having this property for some $M$ gives one for every $M \in \mathbb{Z}$. The GLP takes a quite simple form in the case of the Sierpiński triangle (cf. [20], [П]]). However, in general, it is a rather delicate property. If $l_{M}$ is a good 
labeling function of order $M$ on $V_{M}^{\langle\infty\rangle}$, then it is typically not true that restricting $l_{M}$ to $V_{M+1}^{\langle\infty\rangle}$ gives the GLP of order $M+1$ (see [9], Remark 3.1).

Now, for the unbounded fractal $\mathcal{K}^{\langle\infty\rangle}$ having the GLP, we define a projection map

$$
\mathcal{K}^{\langle\infty\rangle} \ni x \longmapsto \pi_{M}(x) \in \mathcal{K}^{\langle M\rangle}
$$

by the formula

$$
\pi_{M}(x)=R_{\Delta_{M}}\left(x-\sum_{j=M+1}^{J} L^{j} \nu_{i_{j}}\right),
$$

where $\Delta_{M}=\mathcal{K}^{\langle M\rangle}+\sum_{j=M+1}^{J} L^{j} \nu_{i_{j}}$ is an $M$-complex containing $x$, and $R_{\Delta_{M}}$ is the unique rotation determined by $([2.5)$. Here, the two cases are possible:

(1) if $x \notin V_{M}^{\langle\infty\rangle}$, then $\Delta_{M}=\Delta_{M}(x)$ (i.e. $\Delta_{M}$ can be chosen uniquely);

(2) if $x \in V_{M}^{\langle\infty\rangle}$, then $\Delta_{M}$ is one of the $M$-complexes $\Delta_{M}^{(i)}$ such that

$$
\{x\}=\bigcap_{i=1}^{r_{x}} \Delta_{M}^{(i)},
$$

where $r_{x}=\operatorname{rank}(x)$ is the number of $M$-complexes meeting at $x$.

If $x$ is a vertex from $V_{M}^{\langle\infty\rangle}$, possibly belonging to more than one $M$-complex, then indeed we can choose any of those complexes in the definition above - thanks to the GLP of $\mathcal{K}^{\langle\infty\rangle}$; the image does not depend on the particular choice of $\Delta_{M}^{(i)}$.

The projection $\pi_{M}$ is an essential tool to construct the reflected Brownian motion on $\mathcal{K}^{\langle M\rangle}$.

2.3. Reflected Brownian motion on simple nested fractals. We denote by $Z=\left(Z_{t}, \mathbf{P}^{x}\right)_{t \geqslant 0, x \in \mathcal{K}}\langle\infty\rangle$ the Brownian motion on the USNF $\mathcal{K}^{\langle\infty\rangle}$. In the case of the Sierpiński gasket such a process has been rigorously constructed in [3]. For general nested fractals, the Brownian motion has been first constructed on the unit fractal $\mathcal{K}^{\langle 0\rangle}$ ([119], see also [118]) and then extended to $\mathcal{K}^{\langle\infty\rangle}$ by means of Dirichlet forms ([6], see also [15], [22]). It is a strong Markov process with continuous paths whose distributions are invariant under local isometries of $\mathcal{K}^{\langle\infty\rangle}$. It has transition probability densities $g(t, x, y)$ with respect to the $d_{f}$-dimensional Hausdorff measure $\mu$ on $\mathcal{K}^{\langle\infty\rangle}$, i.e.,

$$
\mathbf{P}^{x}\left(Z_{t} \in A\right)=\int_{A} g(t, x, y) \mu(d y), \quad t>0, x \in \mathcal{K}^{\langle\infty\rangle}, A \subset \mathcal{B}\left(\mathcal{K}^{\langle\infty\rangle}\right),
$$

which are jointly continuous on $(0, \infty) \times \mathcal{K}^{\langle\infty\rangle} \times \mathcal{K}^{\langle\infty\rangle}$ and have the scaling property

$$
g(t, x, y)=L^{d_{f}} g\left(L^{d_{w}} t, L x, L y\right), \quad t>0, x, y \in \mathcal{K}^{\langle\infty\rangle} .
$$

Moreover, there are absolute constants $c_{13}, \ldots, c_{16}>0$ such that the following 
sub-Gaussian estimates (see [13], Theorems 5.2 and 5.5) hold true:

$$
\begin{aligned}
& c_{13} t^{-d_{f} / d_{w}} \exp \left(-c_{14}\left(\frac{|x-y|^{d_{w}}}{t}\right)^{1 /\left(d_{J}-1\right)}\right) \leqslant g(t, x, y) \\
\leqslant & c_{15} t^{-d_{f} / d_{w}} \exp \left(-c_{16}\left(\frac{|x-y|^{d_{w}}}{t}\right)^{1 /\left(d_{J}-1\right)}\right), \quad t>0, x, y \in \mathcal{K}^{\langle\infty\rangle} .
\end{aligned}
$$

The constant $d_{J}>1$, called the chemical exponent of $\mathcal{K}^{\langle\infty\rangle}$, is a parameter describing the shortest path scaling of the set $\mathcal{K}^{\langle\infty\rangle}$. Typically, $d_{J} \neq d_{w}$, but it is known that for the Sierpiński gasket one has $d_{J}=d_{w}$. The above estimates were proved under the assumption that there exists $n \in \mathbb{N}$ such that for any $M \in \mathbb{Z}$, if $x, y \in \mathcal{K}^{\langle\infty\rangle}$ satisfy $|x-y| \leqslant L^{M}$, then $d_{M}(x, y) \leqslant n$ (see [13], Section 5). It was shown in [9] that in fact this assumption holds true for any planar simple nested fractal. For a fair account of the theory of Brownian motion on simple nested fractals we refer to [1] and the references therein.

The reflected Brownian motion on $\mathcal{K}^{\langle M\rangle}$ was constructed in [9] as a canonical projection of the free Brownian motion

$$
Z_{t}^{M}=\pi_{M}\left(Z_{t}\right)
$$

Formally, it is the process $Z^{M}=\left(Z_{t}^{M}, \mathbf{P}_{M}^{x}\right)_{t \geqslant 0, x \in \mathcal{K}\langle M\rangle}$, where the measures $\mathbf{P}_{M}^{x}$ are determined by

$$
\mathbf{P}_{M}^{x}\left(Z_{t_{1}}^{M} \in A_{1}, \ldots, Z_{t_{n}}^{M} \in A_{n}\right)=\mathbf{P}^{x}\left(Z_{t_{1}} \in \pi_{M}^{-1}\left(A_{1}\right), \ldots, Z_{t_{n}} \in \pi_{M}^{-1}\left(A_{n}\right)\right)
$$

for every $0 \leqslant t_{1}<t_{2}<\ldots<t_{n}, x \in \mathcal{K}^{\langle M\rangle}$ and $A_{1}, \ldots, A_{n} \in \mathcal{B}\left(\mathcal{K}^{\langle M\rangle}\right)$. As mentioned above, its transition probabilities are absolutely continuous with respect to the measure $\mu$ (restricted to $\mathcal{K}^{\langle M\rangle}$ ) with densities $g_{M}(t, x, y)$ given by

$$
g_{M}(t, x, y)= \begin{cases}\sum_{y^{\prime} \in \pi_{M}^{-1}(y)} g\left(t, x, y^{\prime}\right) & \text { if } y \in \mathcal{K}^{\langle M\rangle} \backslash V_{M}^{\langle M\rangle}, \\ \sum_{y^{\prime} \in \pi_{M}^{-1}(y)} g\left(t, x, y^{\prime}\right) \cdot \operatorname{rank}\left(y^{\prime}\right) & \text { if } y \in V_{M}^{\langle M\rangle}\end{cases}
$$

where $\operatorname{rank}\left(y^{\prime}\right)$ is the number of $M$-complexes meeting at the point $y^{\prime}$. Moreover, as proved in [9], Theorems 4.1 and 4.2, the function $g_{M}(t, x, y)$ is continuous in $(t, x, y)$ and symmetric and bounded in $(x, y)$ for every fixed $t>0$. This provides us with further regularity properties of the process $\left(Z_{t}^{M}\right)_{t \geqslant 0}$ such as the Feller and the strong Feller property.

Our aim in the present paper is to find the sharp two-sided estimates for the densities $g_{M}(t, x, y)$ and for $g_{M}(t, x, y)-g(t, x, y)$. This goal will be achieved in the next section. 


\section{ESTIMATES}

We are now in a position to state our main results in this paper. For given $c>0$ and for every $t>0, M \in \mathbb{Z}$ and $r \geqslant 0$ we put

$$
f_{c}(t, r)=t^{-d_{f} / d_{w}} \exp \left(-c\left(\frac{r^{d_{w}}}{t}\right)^{1 /\left(d_{J}-1\right)}\right)
$$

and

$$
h_{c}(t, M)=L^{-d_{f} M}\left(\frac{L^{M}}{t^{1 / d_{w}}} \vee 1\right)^{d_{f}-d_{w} /\left(d_{J}-1\right)} \exp \left(-c\left(\frac{L^{M}}{t^{1 / d_{w}}} \vee 1\right)^{d_{w} /\left(d_{J}-1\right)}\right) \text {. }
$$

The first theorem gives the sharp two-sided estimates for $g_{M}(t, x, y)$.

THEOREM 3.1. Let $\mathcal{K}\langle\infty\rangle$ be the USNF with the GLP. Then there exist constants $c_{1}, \ldots, c_{6}>0$ such that for every $t>0, M \in \mathbb{Z}$ and $x, y \in \mathcal{K}^{\langle M\rangle}$ we have

$$
\begin{aligned}
c_{1}\left(f_{c_{2}}(t,|x-y|) \vee h_{c_{3}}(t, M)\right) & \leqslant g_{M}(t, x, y) \\
& \leqslant c_{4}\left(f_{c_{5}}(t,|x-y|) \vee h_{c_{6}}(t, M)\right) .
\end{aligned}
$$

We also obtain sharp two-sided bounds for the difference $g_{M}(t, x, y)-g(t, x, y)$.

THEOREM 3.2. Let $\mathcal{K}^{\langle\infty\rangle}$ be the USNF with the GLP. Then there exist constants $c_{7}, \ldots, c_{12}>0$ such that for every $t>0, M \in \mathbb{Z}$ and $x, y \in \mathcal{K}^{\langle M\rangle}$ we have

$$
\begin{aligned}
c_{7}\left(f_{c 8}\left(t, \delta_{M}(x, y)\right) \vee h_{c 9}(t, M)\right) & \leqslant g_{M}(t, x, y)-g(t, x, y) \\
& \leqslant c_{10}\left(f_{c_{11}}\left(t, \delta_{M}(x, y)\right) \vee h_{c_{12}}(t, M)\right),
\end{aligned}
$$

where $\delta_{M}(x, y)=\min _{z \in V_{M}^{*}}(|x-z|+|z-y|)$ with

$$
V_{M}^{*}:=\left\{z \in V_{M}^{\langle M\rangle}: \text { there exists } \Delta_{M} \in \mathcal{T}_{M} \text { such that } \Delta_{M} \cap \mathcal{K}^{\langle M\rangle}=\{z\}\right\} .
$$

We give the proofs of the above theorems after a sequence of auxiliary results. First we fix some useful notation. For $M \in \mathbb{Z}$ and $y \in \mathcal{K}^{\langle M\rangle} \backslash V_{M}^{\langle M\rangle}$ we let

$$
A(M, m, y)=\left\{y^{\prime} \in \pi_{M}^{-1}(y): y^{\prime} \in \mathcal{K}^{\langle M+m+1\rangle} \backslash \mathcal{K}^{\langle M+m\rangle}\right\}, \quad m \geqslant 0,
$$

and

$$
\begin{aligned}
& B(M, 0, y)=\left\{y^{\prime} \in \pi_{M}^{-1}(y): y^{\prime} \in \mathcal{K}^{\langle M+1\rangle} \backslash \mathcal{K}^{\langle M\rangle}, \Delta_{M}\left(y^{\prime}\right) \cap \mathcal{K}^{\langle M\rangle}=\emptyset\right\}, \\
& C(M, 0, y)=\left\{y^{\prime} \in \pi_{M}^{-1}(y): y^{\prime} \in \mathcal{K}^{\langle M+1\rangle} \backslash \mathcal{K}^{\langle M\rangle}, \Delta_{M}\left(y^{\prime}\right) \cap \mathcal{K}^{\langle M\rangle} \neq \emptyset\right\},
\end{aligned}
$$


so that

$$
A(M, 0, y)=B(M, 0, y) \cup C(M, 0, y) .
$$

Then we can decompose the fiber of $y$ as follows:

$$
\pi_{M}^{-1}(y)=\bigcup_{m \geqslant 1} A(M, m, y) \cup B(M, 0, y) \cup C(M, 0, y) \cup\{y\},
$$

and, consequently, for every $x, y \in \mathcal{K}^{\langle M\rangle} \backslash V_{M}^{\langle M\rangle}$, we get

$$
\begin{aligned}
g_{M}(t, x, y)=\underbrace{\sum_{m \geqslant 1} \sum_{y^{\prime} \in A(M, m, y)} g\left(t, x, y^{\prime}\right)+\sum_{y^{\prime} \in B(M, 0, y)} g\left(t, x, y^{\prime}\right)}_{=: g_{M}^{(1)}(t, x, y)} \\
+\underbrace{\underbrace{}_{y^{\prime} \in C(M, 0, y)} g\left(t, x, y^{\prime}\right)}_{=: g_{M}^{(2)}(t, x, y)}+g(t, x, y) .
\end{aligned}
$$

Note that $B(M, 0, y)$ can be an empty set (the only planar example of $\mathcal{K}\langle\infty\rangle$ with this property is the Sierpiński gasket). Here we use the convention that the summation over an empty set always gives zero.

The following lemma will be used in proving our estimates for the function $g_{M}^{(1)}(t, x, y)$. It can be interpreted as the intrinsic growth property of the graph metric with respect to the Euclidean metric.

Lemma 3.1 ([9], Lemma A.2). For every $M \in \mathbb{Z}$ and every $x, y \in \mathcal{K}\langle\infty\rangle$ we have

$$
c_{17} L^{-M}|x-y| \leqslant d_{M}(x, y) \leqslant \max \left\{2, c_{18} N^{-M}|x-y|^{d_{f}}\right\},
$$

where $c_{17}, c_{18}$ are independent of $x, y$ and $M$.

The next two lemmas will be applied to get the upper bounds for the function $g_{M}^{(2)}(t, x, y)$ in the decomposition (B.3)).

LEMMA 3.2. There exists a constant $c_{19}>0$ with the following property. For every $x, y \in \mathcal{K}^{\langle\infty\rangle}$ and $m \in \mathbb{Z}$ such that $x \in \Delta_{m}^{(1)}, y \in \Delta_{m}^{(2)}$ and $\Delta_{m}^{(1)} \cap \Delta_{m}^{(2)}=\emptyset$ we have $|x-y| \geqslant c_{19} L^{m}$.

Pr o o f. The lemma follows from Corollary A.1 in [9] by scaling.

LEMMA 3.3. There exists an absolute constant $c_{20}>0$ with the following property. If $x, y \in \mathcal{K}^{\langle M\rangle}$ and $y^{\prime} \in \pi_{M}^{-1}(y) \backslash\{y\}$ is inside an $M$-complex $\Delta_{M}$ such that $\Delta_{M} \cap \mathcal{K}^{\langle M\rangle}=\{z\}$ for some $z \in V_{M}^{\langle M\rangle}$, then

$$
\left|x-y^{\prime}\right| \geqslant c_{20}(|x-z|+|z-y|) .
$$

In particular, $\left|x-y^{\prime}\right| \geqslant c_{20}|x-y|$. 
Proof. Assume first that $x, y \in \mathcal{K}^{\langle M\rangle} \backslash V_{M}^{\langle M\rangle}$ and let $y^{\prime} \in \pi_{M}^{-1}(y)$ be such that $\Delta_{M}\left(y^{\prime}\right) \cap \mathcal{K}^{\langle M\rangle}=\{z\}$ with some $z \in V_{M}^{\langle M\rangle}$.

Observe that for every $m \leqslant M$ the vertex $z$ lies at the intersection of two $m$ complexes $\Delta_{m}^{(1)}(z)$ and $\Delta_{m}^{(2)}(z)$ such that $\Delta_{m}^{(1)}(z) \subseteq \mathcal{K}^{\langle M\rangle}$ and $\Delta_{m}^{(2)}(z) \nsubseteq \mathcal{K}^{\langle M\rangle}$.

Let now $m \in \mathbb{Z}$ be the smallest integer for which $x, y \in \Delta_{m}^{(1)}(z)$. Then we have $|x-z|,|y-z| \leqslant L^{m} c_{1}^{\prime}$, where $c_{1}^{\prime}$ is the diameter of any zero-complex. On the other hand, as $x \notin \Delta_{m-1}^{(1)}(z)$ or $y \notin \Delta_{m-1}^{(1)}(z)$, we see that $x$ and $y^{\prime}$ are in disjoint $(m-1)$-complexes. Those $(m-1)$-complexes are included in two different $m$-complexes and at most one of them is attached to $z$. Then, from Lemma 3.2 we get $\left|x-y^{\prime}\right| \geqslant L^{m-1} c_{19}$ and, in consequence,

$$
|x-z|+|z-y| \leqslant \frac{2 c_{1}^{\prime} L}{c_{19}}\left|x-y^{\prime}\right|
$$

By continuity of the Euclidean distance, the same is true for every $x, y \in \mathcal{K}^{\langle M\rangle}$. The second assertion follows from the triangle inequality for such a distance. The lemma holds with $c_{20}=c_{19} /\left(2 c_{1}^{\prime} L\right)$.

We now give the estimates for the integral, which will be useful in the proofs of the upper and lower bound in Lemma 3.5 .

LEMMA 3.4. For fixed $\eta, \beta, \gamma>0$ there exist positive constants $c_{21}, c_{22}$ such that for every $a>0$

$$
c_{21}(a \vee 1)^{\beta-\gamma+1} e^{-\eta(a \vee 1)^{\gamma}} \leqslant \int_{a}^{\infty} y^{\beta} e^{-\eta y^{\gamma}} d y \leqslant c_{22}(a \vee 1)^{\beta-\gamma+1} e^{-\eta(a \vee 1)^{\gamma}} .
$$

Pro of. Throughout this proof, constants are independent of $a$ (may depend on $\eta, \beta, \gamma)$. Using the l'Hospital rule, one can see that

$$
\lim _{a \rightarrow \infty} \frac{\int_{a}^{\infty} y^{\beta} e^{-\eta y^{\gamma}} d y}{a^{\beta-\gamma+1} e^{-\eta a^{\gamma}}}=c_{1}^{\prime}>0
$$

so there exists $c_{2}^{\prime}>1$ such that for $a>c_{2}^{\prime}$ we have

$$
\frac{c_{1}^{\prime}}{2} a^{\beta-\gamma+1} e^{-\eta a^{\gamma}} \leqslant \int_{a}^{\infty} y^{\beta} e^{-\eta y^{\gamma}} d y \leqslant \frac{3 c_{1}^{\prime}}{2} a^{\beta-\gamma+1} e^{-\eta a^{\gamma}} .
$$

On the other hand, for $a \in\left[1, c_{2}^{\prime}\right]$ the functions in the numerator and denominator of (3.5) are strictly positive and bounded, therefore there exist $c_{3}^{\prime}, c_{4}^{\prime}>0$ such that for $a \geqslant 1$

$$
c_{3}^{\prime} a^{\beta-\gamma+1} e^{-\eta a^{\gamma}} \leqslant \int_{a}^{\infty} y^{\beta} e^{-\eta y^{\gamma}} d y \leqslant c_{4}^{\prime} a^{\beta-\gamma+1} e^{-\eta a^{\gamma}} .
$$


Finally, as the integrand is bounded for $y \geqslant 0$, one can write

$$
c_{3}^{\prime}(a \vee 1)^{\beta-\gamma+1} e^{-\eta a^{\gamma}} \leqslant \int_{a}^{\infty} y^{\beta} e^{-\eta y^{\gamma}} d y \leqslant c_{5}^{\prime}(a \vee 1)^{\beta-\gamma+1} e^{-\eta a^{\gamma}}
$$

for $a>0$, which completes the proof.

We now give the two-sided bounds for the function $g_{M}^{(1)}(t, x, y)$, which are the first crucial ingredient of the proofs of our main results. This is the case when $y^{\prime}$ under the sums in (B.3) are far away from $x$.

LEMMA 3.5. For every $t>0, M \in \mathbb{Z}$ and $x, y \in \mathcal{K}^{\langle M\rangle} \backslash V_{M}^{\langle M\rangle}$ we have

$$
c_{23} h_{c_{24}}(t, M) \leqslant g_{M}^{(1)}(t, x, y) \leqslant c_{25} h_{c_{26}}(t, M),
$$

with certain numerical constants $c_{23}, \ldots, c_{26}>0$ (independent of $M, t$ and $x, y$ ).

Proof. Let $M \in \mathbb{Z}, t>0$ and $x, y \in \mathcal{K}^{\langle M\rangle} \backslash V_{M}^{\langle M\rangle}$. We now prove the upper bound and the lower bound separately.

The upper bound. Let us observe that for every fixed $m \geqslant 1$ and $y^{\prime} \in$ $A(M, m, y)$ we have $d_{M+m-1}\left(x, y^{\prime}\right)>2$. Then, by applying the upper bound in (B.4) for $d_{M+m-1}\left(x, y^{\prime}\right)$, we get

$$
\left|x-y^{\prime}\right|^{d_{f}} \geqslant \frac{2}{c_{18}} N^{M+m-1} .
$$

Moreover, the number of such points $y^{\prime}$ is equal to the number of $M$-complexes inside $\mathcal{K}^{\langle M+m+1\rangle} \backslash \mathcal{K}^{\langle M+m\rangle}$, i.e. $N^{m}(N-1)$. Analogously, if $y^{\prime} \in B(M, 0, y)$, then $d_{M-1}\left(x, y^{\prime}\right)>2$, which gives

$$
\left|x-y^{\prime}\right|^{d_{f}} \geqslant \frac{2}{c_{18}} N^{M-1} .
$$

There are less than $N-1$ such points.

Then, by using the decomposition of $g_{M}^{(1)}(t, x, y)$ in (3.3), the upper subGaussian estimate for $g\left(t, x, y^{\prime}\right)$ in (‥Z) and the above observations, we get

(3.7) $g_{M}^{(1)}(t, x, y)$

$$
\begin{aligned}
& \leqslant c_{1}^{\prime} t^{-d_{f} / d_{w}} \sum_{m \geqslant 0} N^{m}(N-1) \exp \left(-c_{2}^{\prime}\left(\frac{\left(2 / c_{18}\right)^{d_{w} / d_{f}} N^{(M+m-1) d_{w} / d_{f}}}{t}\right)^{1 /\left(d_{J}-1\right)}\right) \\
& =c_{1}^{\prime} N^{-M+1} t^{-d_{f} / d_{w}} \sum_{m \geqslant 0} N^{M+m-1}(N-1) \exp \left(-c_{3}^{\prime}\left(\frac{N^{(M+m) d_{w} / d_{f}}}{t}\right)^{1 /\left(d_{J}-1\right)}\right),
\end{aligned}
$$

with an appropriate absolute positive constant $c_{3}^{\prime}$. 
Since for any $\eta, \gamma>0$ we have

$$
\int_{N^{M-1}}^{\infty} e^{-\eta \xi^{\gamma}} d \xi=\sum_{m=0}^{\infty} \int_{N^{M-1+m}}^{N^{M+m}} e^{-\eta \xi^{\gamma}} d \xi \geqslant \sum_{m=0}^{\infty} N^{M+m-1}(N-1) e^{-\eta N^{(M+m) \gamma}},
$$

(B.7) can be estimated by an appropriate integral. We then get

$$
\begin{aligned}
g_{M}^{(1)}(t, x, y) & \leqslant \frac{c_{4}^{\prime}}{L^{M d_{f}} t^{d_{f} / d_{w}}} \int_{N^{M-1}}^{\infty} \exp \left(-c_{3}^{\prime}\left(\frac{\xi^{d_{w} / d_{f}}}{t}\right)^{1 /\left(d_{J}-1\right)}\right) d \xi \\
& =\frac{c_{4}^{\prime}}{L^{M d_{f}} t^{d_{f} / d_{w}}} \int_{N^{M-1}}^{\infty} \exp \left(-c_{3}^{\prime}\left(\frac{\xi^{1 / d_{f}}}{t^{1 / d_{w}}}\right)^{d_{w} /\left(d_{J}-1\right)}\right) d \xi
\end{aligned}
$$

which, by the substitution $\xi^{1 / d_{f}} t^{-1 / d_{w}}=\zeta$, is equal to

$$
\frac{c_{4}^{\prime} d_{f}}{L^{M d_{f}}} \int_{N^{(M-1) / d_{f}} t_{t^{-1 / d_{w}}}^{\infty}} \zeta^{d_{f}-1} \exp \left(-c_{3}^{\prime} \zeta^{d_{w} /\left(d_{J}-1\right)}\right) d \zeta
$$

Now, using Lemma 3.4 and the fact that $N^{M / d_{f}}=L^{M}$, we can conclude the proof of the upper bound in (B.6), getting

$$
\begin{aligned}
& g_{M}^{(1)}(t, x, y) \\
\leqslant & c_{5}^{\prime} L^{-d_{f} M}\left(\frac{L^{M-1}}{t^{1 / d_{w}}} \vee 1\right)^{d_{f}-d_{w} /\left(d_{J}-1\right)} \exp \left(-c_{3}^{\prime}\left(\frac{L^{M-1}}{t^{1 / d_{w}}} \vee 1\right)^{d_{w} /\left(d_{J}-1\right)}\right) \\
\leqslant & c_{6}^{\prime} L^{-d_{f} M}\left(\frac{L^{M}}{t^{1 / d_{w}}} \vee 1\right)^{d_{f}-d_{w} /\left(d_{J}-1\right)} \exp \left(-c_{7}^{\prime}\left(\frac{L^{M}}{t^{1 / d_{w}}} \vee 1\right)^{d_{w} /\left(d_{J}-1\right)}\right),
\end{aligned}
$$

which completes the proof of the upper bound.

The lower bound. First recall that $B(M, 0, y)$ can be an empty set. Therefore, we first write

$$
g_{M}^{(1)}(t, x, y) \geqslant \sum_{m \geqslant 1} \sum_{y^{\prime} \in A(M, m, y)} g\left(t, x, y^{\prime}\right) .
$$

When $m \geqslant 1$ and $y^{\prime} \in A(M, m, y)$, we have $d_{M+m+1}\left(x, y^{\prime}\right)=1$. By applying the lower estimate in (B.4) with $n=M+m+1$, we get

$$
\left|x-y^{\prime}\right| \leqslant \frac{1}{c_{17}} L^{M+m+1}
$$


(recall also that the cardinality of $A(M, m, y)$ is equal to $N^{m}(N-1)$ ). Therefore, by the lower sub-Gaussian bound of $g\left(t, x, y^{\prime}\right)$, the series on the right-hand side of (3.8) is larger than or equal to

$$
\begin{aligned}
& c_{8}^{\prime} t^{-d_{f} / d_{w}} \sum_{m \geqslant 1} N^{m}(N-1) \exp \left(c_{9}^{\prime}\left(\frac{\left(1 / c_{17}\right)^{d_{w}} L^{(M+m+1) d_{w}}}{t}\right)^{1 /\left(d_{J}-1\right)}\right) \\
= & c_{8}^{\prime} N^{-M} t^{-d_{f} / d_{w}} \sum_{m \geqslant 1} N^{M+m}(N-1) \exp \left(c_{10}^{\prime}\left(\frac{N^{(M+m) d_{w} / d_{f}}}{t}\right)^{1 /\left(d_{J}-1\right)}\right),
\end{aligned}
$$

where $c_{8}^{\prime}$ and $c_{10}^{\prime}$ are absolute constants. Now, estimating the series by an appropriate integral (as in the proof of the upper bound), we show that the above member is greater than or equal to

$$
\begin{aligned}
c_{11}^{\prime} L^{-M d_{f}} & t^{-d_{f} / d_{w}} \int_{N^{M+1}}^{\infty} \exp \left(-c_{10}^{\prime}\left(\frac{\xi^{d_{w} / d_{f}}}{t}\right)^{1 /\left(d_{J}-1\right)}\right) d \xi \\
= & c_{11}^{\prime} L^{-M d_{f}} t^{-d_{f} / d_{w}} \int_{N^{M+1}}^{\infty} \exp \left(-c_{10}^{\prime}\left(\frac{\xi^{1 / d_{f}}}{t^{1 / d_{w}}}\right)^{d_{w} /\left(d_{J}-1\right)}\right) d \xi \\
& =c_{11}^{\prime} d_{f} L^{-M d_{f}} \int_{L^{M+1} t^{-1 / d_{w}}}^{\infty} \zeta^{d_{f}-1} \exp \left(-c_{10}^{\prime} \zeta^{d_{w} /\left(d_{J}-1\right)}\right) d \zeta
\end{aligned}
$$

Using Lemma B.4, we can now write

$$
\begin{aligned}
& g_{M}^{(1)}(t, x, y) \\
\geqslant & c_{11}^{\prime} L^{-d_{f} M}\left(\frac{L^{M+1}}{t^{1 / d_{w}}} \vee 1\right)^{d_{f}-d_{w} /\left(d_{J}-1\right)} \exp \left(-c_{10}^{\prime}\left(\frac{L^{M+1}}{t^{1 / d_{w}}} \vee 1\right)^{d_{w} /\left(d_{J}-1\right)}\right) \\
\geqslant & c_{12}^{\prime} L^{-d_{f} M}\left(\frac{L^{M}}{t^{1 / d_{w}}} \vee 1\right)^{d_{f}-d_{w} /\left(d_{J}-1\right)} \exp \left(-c_{13}^{\prime}\left(\frac{L^{M}}{t^{1 / d_{w}}} \vee 1\right)^{d_{w} /\left(d_{J}-1\right)}\right) .
\end{aligned}
$$

This completes the proof of the lemma.

We are now ready to give the proofs of our main theorems.

Proof of $\mathrm{T}$ he ore $\mathrm{m}$ 3.1. Let $M \in \mathbb{Z}, t>0$ and assume first that $x, y \in$ $\mathcal{K}^{\langle M\rangle} \backslash V_{M}^{\langle M\rangle}$. Recall that from (3.3) we have

$$
g_{M}(t, x, y)=g_{M}^{(1)}(t, x, y)+g_{M}^{(2)}(t, x, y)+g(t, x, y)
$$


By the sub-Gaussian upper estimate in (2.7) and Lemma B.3, we have

$$
\begin{aligned}
0 \leqslant g_{M}^{(2)}(t, x, y) & \leqslant c_{1}^{\prime} \sum_{y^{\prime} \in C(M, 0, y)} t^{-d_{f} / d_{w}} \exp \left(-c_{2}^{\prime}\left(\frac{\left|x-y^{\prime}\right|^{d_{w}}}{t}\right)^{1 /\left(d_{J}-1\right)}\right) \\
& \leqslant c_{1}^{\prime} k t^{-d_{f} / d_{w}} \exp \left(-c_{2}^{\prime}\left(\frac{\left(c_{20}|x-y|\right)^{d_{w}}}{t}\right)^{1 /\left(d_{J}-1\right)}\right) \\
& =c_{3}^{\prime} t^{-d_{f} / d_{w}} \exp \left(-c_{4}^{\prime}\left(\frac{|x-y|^{d_{w}}}{t}\right)^{1 /\left(d_{J}-1\right)}\right)
\end{aligned}
$$

If $x, y \in \mathcal{K}^{\langle M\rangle} \backslash V_{M}^{\langle M\rangle}$, then the claimed two-sided bounds in Theorem 13.1 follow from a combination of the estimates of $g_{M}^{(1)}$ in Lemma [3.5, the above estimates of $g_{M}^{(2)}$ and the sub-Gaussian two-sided estimates of $g$ in (2.7). By continuity of the function $g_{M}(t, x, y)$ (see [9], Theorem 4.1(1)), these bounds also extend to arbitrary $x, y \in \mathcal{K}^{\langle M\rangle}$. This completes the proof of the theorem.

Proof of Theorem B.2. Let $M \in \mathbb{Z}, t>0$ and let $x, y \in \mathcal{K}^{\langle M\rangle} \backslash V_{M}^{\langle M\rangle}$. As above, we have

$$
g_{M}(t, x, y)-g(t, x, y)=g_{M}^{(1)}(t, x, y)+g_{M}^{(2)}(t, x, y)
$$

by (B.3), and from Lemma 3.5 we obtain

$$
c_{23} h_{c_{24}}(t, M) \leqslant g_{M}^{(1)}(t, x, y) \leqslant c_{25} h_{c_{26}}(t, M) .
$$

It is then enough to estimate $g_{M}^{(2)}(t, x, y)$. From (2.7) and Lemma 3.3 we see that for $y^{\prime} \in C(M, m, y)$

$$
g\left(t, x, y^{\prime}\right) \leqslant c_{1}^{\prime} f_{c_{2}^{\prime}}(|x-z|+|z-y|),
$$

with $z \in V_{M}^{\langle M\rangle}$ such that $\Delta_{M}\left(y^{\prime}\right) \cap \mathcal{K}^{\langle M\rangle}=\{z\}$ and with the absolute constants $c_{1}^{\prime}, c_{2}^{\prime}$. On the other hand, $\left|x-y^{\prime}\right| \leqslant|x-z|+\left|z-y^{\prime}\right|=|x-z|+|z-y|$, which gives

$$
g\left(t, x, y^{\prime}\right) \geqslant c_{3}^{\prime} f_{c_{4}^{\prime}}(|x-z|+|z-y|) .
$$

Then, summing over $y^{\prime} \in C(M, m, y)$, we obtain

$$
\begin{aligned}
g_{M}^{(2)}(t, x, y)=\sum_{y^{\prime} \in C(M, m, y)} g\left(t, x, y^{\prime}\right) & \leqslant c_{1}^{\prime} \sum_{z \in V_{M}^{*}} f_{c_{2}^{\prime}}(t,|x-z|+|z-y|) \\
& \leqslant c_{1}^{\prime} k f_{c_{2}^{\prime}}\left(t, \delta_{M}(x, y)\right)
\end{aligned}
$$


and

$$
\begin{aligned}
g_{M}^{(2)}(t, x, y)=\sum_{y^{\prime} \in C(M, m, y)} g\left(t, x, y^{\prime}\right) & \geqslant c_{3}^{\prime} \sum_{z \in V_{M}^{*}} f_{c_{4}^{\prime}}(t,|x-z|+|z-y|) \\
& \geqslant c_{3}^{\prime} f_{c_{4}^{\prime}}\left(t, \delta_{M}(x, y)\right),
\end{aligned}
$$

where $\delta_{M}(x, y)=\inf _{z \in V_{M}^{*}}(|x-z|+|z-y|)$. As the functions on both sides of (B.10) are continuous in $(x, y)$ on $\mathcal{K}^{\langle M\rangle} \times \mathcal{K}^{\langle M\rangle}$, the above bounds in fact extend to all $x, y \in \mathcal{K}^{\langle M\rangle}$. This completes the proof.

In the sequel, we will write $a(t, x, y, M) \approx b(t, x, y, M)$ if there exist positive constants $c_{1}^{\prime}, c_{2}^{\prime}$ independent of $x, y, t, M$ such that

$$
c_{1}^{\prime} a(t, x, y, M) \leqslant b(t, x, y, M) \leqslant c_{2}^{\prime} a(t, x, y, M) .
$$

We will now describe the behavior of $g_{M}(t, x, y)$ in various time-space regimes. Recall that we have $|x-y| \leqslant L^{M} \operatorname{diam}\left(\mathcal{K}^{\langle 0\rangle}\right)$ for every $x, y \in \mathcal{K}^{\langle M\rangle}$ with $M \in \mathbb{Z}$.

COROLlary 3.1. For every $M \in \mathbb{Z}$ and $x, y \in \mathcal{K}^{\langle M\rangle}$ we have the following. If $t>L^{M d_{w}}$, then

$$
g_{M}(t, x, y) \approx L^{-M d_{f}},
$$

and if $0<t \leqslant L^{M d_{w}}$, then

$$
c_{27} f_{c_{28}}(t,|x-y|) \leqslant g_{M}(t, x, y) \leqslant c_{29} f_{c_{30}}(t,|x-y|),
$$

with certain numerical constants $c_{27}, \ldots, c_{30}>0$ independent of $t, x, y$ and $M$. In particular, for $0<t \leqslant L^{M d_{w}}$ such that $t>|x-y|^{d_{w}}$ we have $g_{M}(t, x, y) \approx$ $t^{-d_{f} / d_{w}}$.

Proof. The first assertion follows directly from the fact that for $t>L^{M d_{w}}$ we have

$$
t^{-d_{f} / d_{w}}<L^{-M d_{f}} \quad \text { and } \quad c_{1}^{\prime} \frac{|x-y|}{t^{1 / d_{w}}} \leqslant \frac{L^{M}}{t^{1 / d_{w}}}<1 .
$$

Indeed, the last two inequalities give

$$
f_{c_{2}^{\prime}}(t,|x-y|) \approx t^{-d_{f} / d_{w}} \quad \text { and } \quad h_{c_{3}^{\prime}}(t, M) \approx L^{-M d_{f}}
$$

and from the estimates in Theorem 3.1 we get $g_{M}(t, x, y) \approx L^{-M d_{f}}$.

Consider now the case $|x-y|^{d_{w}}<t \leqslant L^{M d_{w}}$. Then again $|x-y| / t^{1 / d_{w}} \leqslant 1$, which yields

$$
f_{c_{4}^{\prime}}(t,|x-y|) \approx t^{-d_{f} / d_{w}}
$$

Moreover,

$$
0 \leqslant h_{c_{5}^{\prime}}(t, M)=t^{-d_{f} / d_{w}} a^{-1} \exp \left(-c_{5}^{\prime} a\right)
$$


where $a=\left(L^{M} / t^{1 / d_{w}}\right)^{d_{w} /\left(d_{J}-1\right)} \geqslant 1$. As the function $a \mapsto a^{-1} \exp \left(-c_{5}^{\prime} a\right)$ is bounded for $a \geqslant 1$, we conclude that

$$
f_{c_{6}^{\prime}}(t,|x-y|) \vee h_{c_{5}^{\prime}}(t, M) \approx t^{-d_{f} / d_{w}} .
$$

This also implies (B.TI).

Finally, if $0<t \leqslant L^{M d_{w}}$ and $t \leqslant|x-y|^{d_{w}}$, then by $|x-y| \leqslant c_{7}^{\prime} L^{M}$ and $L^{M} / t^{1 / d_{w}}>1$, we see that

$$
\begin{aligned}
0 \leqslant h_{c_{8}^{\prime}}(t, M) & =t^{-d_{f} / d_{w}}\left(\frac{L^{M}}{t^{1 / d_{w}}}\right)^{-d_{w} /\left(d_{J}-1\right)} \exp \left(-c_{8}^{\prime}\left(\frac{L^{M}}{t^{1 / d_{w}}}\right)^{d_{w} /\left(d_{J}-1\right)}\right) \\
& \leqslant t^{-d_{f} / d_{w}} \exp \left(-c_{9}^{\prime}\left(\frac{|x-y|}{t^{1 / d_{w}}}\right)^{d_{w} /\left(d_{J}-1\right)}\right)=f_{c_{9}^{\prime}}(t,|x-y|) .
\end{aligned}
$$

This again implies (B.]) and completes the proof.

Note that a similar result can be given for the difference $g_{M}(t, x, y)-g(t, x, y)$.

Acknowledgments. The author would like to thank Kamil Kaleta for drawing the attention to the subject and many valuable remarks. The author is grateful to the referee, whose comments helped us to improve the presentation of the paper.

\section{REFERENCES}

[1] M. T. Barlow, Diffusion on fractals, in: Lectures on Probability Theory and Statistics: École d'Été de Probabilités de Saint-Flour XXV - 1995, Lecture Notes in Math., Vol. 1690, Springer, Berlin 1998.

[2] M. T. Barlow and R. F. Bass, The construction of Brownian motion on the Sierpinski carpet, Ann. Inst. Henri Poincaré Probab. Stat. 25 (3) (1989), pp. 225-257.

[3] M. T. Barlow and E. A. Perkins, Brownian motion on the Sierpinski gasket, Probab. Theory Related Fields 79 (4) (1988), pp. 543-623.

[4] K. Bogdan, A. Stós, and P. Sztonyk, Harnack inequality for stable processes on d-sets, Studia Math. 158 (2) (2003), pp. 163-198.

[5] P. J. Fitzsimmons, B. M. Hambly, and T. Kumagai, Transition density estimates for Brownian motion on affine nested fractals, Comm. Math. Phys. 165 (3) (1994), pp. 595-620.

[6] M. Fukushima, Dirichlet forms, diffusion processes and spectral dimensions for nested fractals, in: Ideas and Methods in Mathematical Analysis, Stochastics, and Applications. In Memory of R. Hoegh-Krohn, Vol. 1, S. Albeverio et al. (Eds.), Cambridge University Press, Cambridge 1992, pp. 151-161.

[7] S. Goldstein, Random walks and diffusions on fractals, in: Percolation Theory and Ergodic Theory of Infinite Particle Systems, IMA Vol. Math Appl., Vol. 8, Springer, New York 1987, pp. 121-128.

[8] K. Kaleta and M. Kwaśnicki, Boundary Harnack inequality for $\alpha$-harmonic functions on the Sierpiński triangle, Probab. Math. Statist. 30 (2) (2010), pp. 353-368.

[9] K. Kaleta, M. Olszewski, and K. Pietruska-Pałuba, Reflected Brownian motion on simple nested fractals, Fractals 27 (6) (2019), art. 1950104.

[10] K. Kaleta and K. Pietruska-Pałuba, Integrated density of states for PoissonSchrödinger perturbations of subordinate Brownian motions on the Sierpinski gasket, Stochastic Process. Appl. 125 (4) (2015), pp. 1244-1281. 
[11] K. Kaleta and K. Pietruska-Pałuba, Lifschitz singularity for subordinate Brownian motions in presence of the Poissonian potential on the Sierpinski gasket, Stochastic Process. Appl. 128 (11) (2018), pp. 3897-3939.

[12] J. Kigami, Analysis on Fractals, Cambridge University Press, Cambridge 2001.

[13] T. Kumagai, Estimates of transition densities for Brownian motion on nested fractals, Probab. Theory Related Fields 96 (2) (1993), pp. 205-224.

[14] T. Kumagai, Brownian motion penetrating fractals: An application of the trace theorem of Besov spaces, J. Funct. Anal. 170 (1) (2000), pp. 69-92.

[15] T. Kumagai and S. Kusuoka, Homogenization on nested fractals, Probab. Theory Related Fields 104 (3) (1996), pp. 375-398.

[16] T. Kumagai and K. Sturm, Construction of diffusion processes on fractals, d-sets, and general metric measure spaces, J. Math. Kyoto Univ. 45 (2) (2005), pp. 307-327.

[17] S. Kusuoka, A diffusion process on a fractal, in: Probabilistic Methods in Mathematical Physics: Proceedings of the Taniguchi International Symposium, Katata and Kyoto, 1985, Kino Kuniya-North Holland, Amsterdam 1987, pp. 251-274.

[18] S. Kusuoka, Dirichlet forms on fractals and products of random matrices, Publ. Res. Inst. Math. Sci. 25 (4) (1989), pp. 659-680.

[19] T. Lindstrøm, Brownian Motion on Nested Fractals, Mem. Amer. Math. Soc. 83 (420) (1990).

[20] K. Pietruska-Pałuba, The Lifschitz singularity for the density of states on the Sierpinski gasket, Probab. Theory Related Fields 89 (1) (1991), pp. 1-33.

[21] K. Pietruska-Pałuba, The Wiener sausage asymptotics on simple nested fractals, Stoch. Anal. Appl. 23 (1) (2005), pp. 111-135.

[22] T. Shima, Lifschitz tails for random Schrödinger operators on nested fractals, Osaka J. Math. 29 (4) (1992), pp. 749-770.

[23] R. S. Strichartz, Analysis on fractals, Notices Amer. Math. Soc. 46 (10) (1999), pp. 11991208.

[24] R. S. Strichartz, Differential Equations on Fractals: A Tutorial, Princeton University Press, Princeton, NJ, 2006.

Mariusz Olszewski

Faculty of Pure and Applied Mathematics

Wrocław University of Science and Technology

Wybrzeże Wyspiańskiego 27

50-370 Wrocław, Poland

E-mail: mariusz.olszewski@pwr.edu.pl

Received on 7.3.2018;

revised version on 28.6.2018 